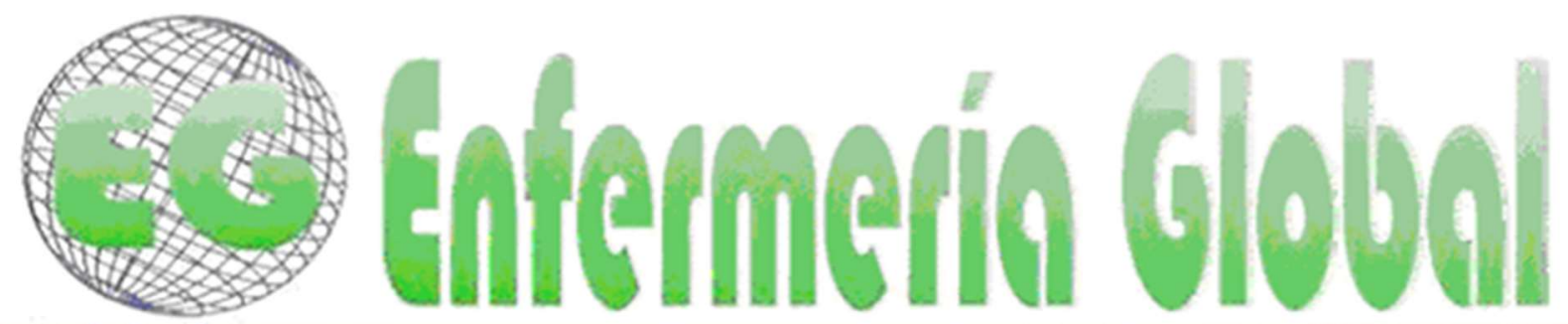

\title{
ORIGINALES
}

\section{Necesidades en familiares de pacientes críticos de una institución de IV nivel en Montería, Colombia}

Needs in relatives of critical patients of a IV level institution in Monteria, Colombia

\author{
Jorge Luis Herrera Herrera ${ }^{1}$ \\ Yolima Judith Llorente Pérez ${ }^{2}$ \\ Sadith José Suarez Mendoza ${ }^{3}$ \\ Edinson Oyola López ${ }^{4}$
}

1 Enfermero. Magister en Enfermería. Jefe de Área de Investigación. Universidad del Sinú Elías Bechara Zainúm. Montería. Córdoba. Colombia. jlherrera2201@gmail.com

2 Enfermera. Magister en Salud Pública, Docente Investigadora. Universidad del Sinú Elías Bechara Zainúm. Montería. Córdoba. Colombia.

${ }^{3}$ Enfermero. Universidad del Sinú Elías Bechara Zainúm. Montería. Córdoba. Colombia.

4 Enfermero. Magíster en Enfermería. Coordinador de enfermería en centro de cuidado renal Davita. Montería. Córdoba. Colombia.

\section{https://doi.org/10.6018/eglobal.423121}

Recibido: $15 / 04 / 2020$

Aceptado: 4/09/2020

\section{RESUMEN:}

Objetivo: Determinar las necesidades en familiares de pacientes críticos de una institución de IV nivel en Montería, Colombia.

Metodología: Investigación descriptiva, transversal con enfoque cuantitativo. Para la recolección de la información se aplicó el Cuestionario de Necesidades de los Familiares de Pacientes de Cuidados Intensivos y una cédula de datos sociodemográficos.

Resultados: Las necesidades que se determinaron fueron la información sincera respecto al estado y progreso del paciente y recibir explicación del equipamiento que está utilizándose. La dimensión que presentó mayores necesidades fue la de comunicación.

Conclusiones: El familiar de una persona ingresada en un servicio de cuidado intensivo debe ser tomado en cuenta en el proceso de atención.

Palabras clave: Evaluación Necesidades; Cuidadores; Cuidado Crítico.

\section{ABSTRACT:}

Objective: To determine the needs in relatives of critically ill patients of an IV level institution in Montería, Colombia.

Methodology: Descriptive, cross-sectional research with a quantitative approach. For the collection of information, the Questionnaire of Needs of the Relatives of Intensive Care Patients and a sociodemographic data card were applied.

Results: The needs that were determined were honest information regarding the state and progress of the patient and receive an explanation of the equipment being used. The dimension that presented the greatest needs was that of communication. 
Conclusions: The family of a person admitted to an intensive care service should be taken into account in the care process.

Keywords: Needs Assessment; Caregivers; Critical Care

\section{INTRODUCCIÓN}

Las unidades de cuidados intensivos (UCI), se caracterizan por ser servicios de alta complejidad en la atención, dada las patologías de los pacientes y por las tecnologías y cuidados que se requieren para el restablecimiento de las funciones corporales, tienen como objetivo garantizar un cuidado integral a la persona que se encuentra en una situación de desequilibrio fisiológico, y que dicha alteración puede llevarlo a padecer complicaciones y/o la muerte. (1)

En ese sentido, al hablar de dichas unidades funcionales, de su estructura y complejidad, se debe tener en cuenta tanto al paciente como a sus familiares, dado que la internación de un miembro de la familia en dichos servicios puede tener resonancia en la dinámica familiar, y también pueden verse sometidos a una serie de eventos como angustia, estrés, ansiedad, entre otros, derivados del diagnóstico, tratamiento, pronóstico, cura, complicación o muerte o por las dificultades en la comunicación entre el paciente y familia. ${ }^{(1)}$

De esta forma, se tiene que la internación en $\mathrm{UCl}$ puede generar temores tanto en el paciente como en la familia, y en estos últimos, sobre todo en las visitas iniciales, que pueden ser alarmantes, crean angustia, expectativas en relación con la cantidad de dispositivos de monitoreo, equipos de mantenimiento de la vida y controles ambientales necesarios para garantizar un cuidado con calidad. (2)

En ese orden de ideas, los familiares de los pacientes hospitalizados en dicha unidad funcional, también precisan de cuidados para preservar su salud física y mental, requiriendo establecer una estrecha relación con alguien a quien poder confiar sus necesidades, dificultades, preocupaciones o dolencias, dado que en el entorno de $\mathrm{UCl}$ no solo deben figurar como visitantes, sino como una extensión del paciente, que experimentan el proceso de cuidado al lado de su familiar ${ }^{(3)}$.

Desde esa óptica, el familiar requiere evaluación de sus necesidades, las cuales pueden ser con respecto a seguridad, información, entre otras, y los miembros del equipo de salud, específicamente médico o enfermería ${ }^{(3)}$, pueden garantizar dicha atención.

Ante las necesidades de los familiares, la evidencia relaciona que estos consideran importante el suministro de información con respecto al pronóstico o condición clínica de su paciente ${ }^{(3)}$ y efectividad del tratamiento realizado ${ }^{(4)}$. Así mismo, consideran que se hace necesario que siempre se les hable con la verdad, que la evolución del paciente sea otorgada con datos muy específicos ${ }^{(4)}$, solicitan cuidados integrales y de calidad, que la atención no genere daño al paciente, que se disminuyan los riesgos (5), así mismo solicitan mayor proximidad con su familiar y satisfacción de necesidades emocionales, sociales y prácticas ${ }^{(6,7)}$.

Por su parte, Padilla et al ${ }^{(8)}$, en un estudio realizado en Chile, identificaron que los factores económicos o financieros suelen ser otra necesidad de los familiares, así 
como la comunicación. De igual forma, Martos - Casado (9) en una investigación realizada en España, señala que los familiares requieren suplir necesidades de información, dado que la información suministrada no es comprendida en su totalidad.

En Colombia, específicamente en el departamento de Córdoba se han realizado estudios referentes al fenómeno antes mencionado y sugieren seguir investigándolo, con la finalidad de favorecer el vínculo paciente - familia - equipo de salud y recomiendan educar al personal de salud sobre las necesidades más importantes que tienen los familiares frente a una internación en $\mathrm{UCl}$.

Teniendo en cuenta lo descrito, surge la siguiente pregunta de investigación ¿Cuáles son las necesidades en familiares de pacientes críticos de una institución de IV nivel en Montería, Colombia?

La identificación de las necesidades puede servir de herramienta para mejorar los procesos de atención en salud, los cuales se deben focalizar en la diada paciente familia, toda vez que un ingreso a $\mathrm{UCl}$ implica afectaciones de diversa índole.

La familia debe ser abordada de forma integral, y para ello se requiere que los profesionales de la salud conozcan sus necesidades y las herramientas necesarias para ayudarlos en la carga que genera dicho proceso.

En ese abordaje integral, enfermería juega un papel fundamental como disciplina humanística, que se adhiere a una filosofía básica centrada en el ser humano y su interacción con el entorno, y es el nexo entre la familia y los demás integrantes del equipo de salud, por su permanencia continua en el servicio, que permite ayudar al paciente y familia a adaptarse a su nueva forma de vida, favoreciendo así su rehabilitación integral. (2)

\section{OBJETIVOS}

- Determinar las necesidades en familiares de pacientes críticos de una institución de IV nivel en Montería, Colombia.

- Caracterizar la población según variables de interés: género, edad, nivel de escolaridad y parentesco.

- Identificar las dimensiones que presentan mayores necesidades.

\section{MATERIAL Y MÉTODO}

\section{Tipo de estudio}

Investigación de tipo descriptiva, transversal, cuyo propósito fue la identificación de las necesidades de los familiares de pacientes hospitalizados en una Unidad de Cuidados Intensivos (UCI), adscrita a una institución de IV nivel de complejidad de la ciudad de Montería; los cuales asistían a la visita durante los meses de octubre a diciembre de 2019; periodo que demoró la recolección de la información. En atención al tratamiento de los datos, corresponde a un diseño con enfoque cuantitativo, El instrumento utilizado fue el Cuestionario de Necesidades de los Familiares de 
Pacientes de Cuidados Intensivos, el cual cuenta con pruebas de validez que recomiendan su uso ${ }^{(10)}$.

\section{Unidad de análisis}

La unidad de análisis estuvo conformada por las necesidades de los familiares que participaron en el estudio, así como las variables sociodemográficas que se seleccionaron para caracterizar a la muestra.

\section{Muestra y muestreo}

La muestra la conformaron 340 cuidadores familiares, que asistieron a la visita durante el tiempo que demoró la fase de recolección de la información. El muestreo fue de tipo intencional, teniendo en cuenta los criterios de inclusión y exclusión establecidos.

En este sentido, se incluyeron en el estudio personas mayores de edad, firmantes del consentimiento informado institucional parar el ingreso a $\mathrm{UCl}$ de su paciente; lo que los acreditaba como el familiar responsable. Así mismo, un criterio de inclusión era asistir todos los días a la visita y fueron excluidos los reingresos antes de las 48 horas, y aquellos familiares que no asistían de forma continua al pase de visita.

\section{Instrumentos}

El instrumento utilizado para medir las necesidades de la muestra seleccionada fue el Cuestionario de Necesidades de los Familiares de Pacientes de Cuidados Intensivos (CCFNI) - versión breve; el cual ha sido validado en idioma castellano por Gómez et al, quien determinó un a de Cronbach general de 0,655. (10)

Contiene 11 preguntas y sus respuestas son en escala tipo Likert, con rango 1- 4 (1= Casi todas las veces; 2= La mayoría de las veces; 3= Solo algunas veces; 4= Nunca). La escala arroja una puntuación general, mínima de 11 y máxima de 44 puntos, cuanto mayor sea la puntuación, más necesidades percibidas. Internamente el instrumento está compuesto por 4 subescalas, cada una de estas con puntuación mínima y máxima: atención médica, comunicación, atención personal y posibles mejoras. Las tres primeras tienen una puntuación mínima de 3 y máxima de 12, la última subescala tiene una mínima puntuación de 2 y máxima de 8 .

Para la caracterización sociodemográfica se diseñó una cédula de datos, que incluía las variables de género, edad, nivel de escolaridad y parentesco con el paciente. 


\section{RESULTADOS}

Tabla 1. Características sociodemográficas de los familiares

\begin{tabular}{llcc}
\hline Variable & & $\boldsymbol{f}$ & $\%$ \\
\hline Género & Masculino & 160 & 47 \\
& Femenino & 180 & 53 \\
Rango de edad & $18-29$ & 51 & 15 \\
(años) & $30-49$ & 119 & 35 \\
& $50-69$ & 153 & 45 \\
Grado de & 70 o más & 17 & 5 \\
escolaridad & Primaria completa & 153 & 45 \\
& Bachillerato Completo & 102 & 30 \\
Parentesco & Universitario & 85 & 25 \\
& Hijo (a) & 204 & 60 \\
& Esposo (a) & 102 & 30 \\
& Otro & 34 & 10 \\
\hline
\end{tabular}

Fuente: cedula de datos sociodemográficos

La caracterización sociodemográfica, permitió establecer que los familiares eran en su mayoría mujeres en edades entre 50 y 69 años de edad, con grado de escolaridad de bachillerato completo y el parentesco que más se encontró fue ser hijo (a). De la anterior caracterización, llama la atención como dato relevante que toda la muestra presentó algún grado de escolaridad, tal como se muestra en la Tabla 1.

Tabla 2. Necesidades de los familiares

\begin{tabular}{llll}
\hline Orden & \multicolumn{1}{c}{ Necesidad } & Dimensión & Media +/- DE \\
\hline 1 & $\begin{array}{l}\text { Información sincera respecto al } \\
\text { estado y progreso del paciente }\end{array}$ & Comunicación & $3,98+/-0,18$ \\
2 & $\begin{array}{l}\text { Explicación del equipamiento } \\
\text { que está utilizándose }\end{array}$ & Comunicación & $3,95+/-0,13$ \\
3 & $\begin{array}{l}\text { Interés por parte del equipo, de } \\
\text { cómo está usted. }\end{array}$ & Atención personal & $3,93+/-0,16$ \\
4 & $\begin{array}{l}\text { Satisfacción con las atenciones } \\
\text { médicas recibidas por el }\end{array}$ & Atención medica & $3,88+/-0,28$ \\
& $\begin{array}{l}\text { paciente la sala de } \\
\text { Comodidad de lasibles mejoras }\end{array}$ & $3,86+/-0,25$ \\
& espera & & \\
\hline
\end{tabular}

Fuente: (CCFNI) 
Al momento de revisar las principales necesidades identificadas por los familiares, se pudo establecer que cinco fueron las que obtuvieron mayor puntuación por parte de ellos y en su orden, de acuerdo a la media de los datos obtenidos fueron: Información sincera respecto al estado y progreso del paciente $(\bar{X}=3,98 ; D E=0,18)$, explicación del equipamiento que está utilizándose $(\bar{X}=3,95 ; D E=0,13)$, interés por parte del equipo, de cómo está usted $(\bar{X}=3,93 ; D E=0,16)$, satisfacción con las atenciones médicas recibidas por el paciente $(\bar{X}=3,88$; $D E=0,28)$, y comodidad de la sala de espera $(\bar{X}=3,86 ; D E=0,25)$. Las dos primeras necesidades pertenecían a la dimensión de comunicación, como se indica en la Tabla 2.

Tabla 3. Dimensiones con mayores necesidades

\begin{tabular}{lcc}
\hline Orden & Dimensión & Media +/- DE \\
\hline 1 & Comunicación & $11,4+/-0,15$ \\
2 & Atención personal & $11,2+/-0,12$ \\
3 & Atención medica & $10,3+/-0,19$ \\
4 & Posibles mejoras & $7,6+/-0,14$ \\
\hline \multicolumn{2}{l}{ Puntaje global } & $41,6+/-1,8$ \\
\hline
\end{tabular}

Fuente: (CCFNI)

Respecto a las dimensiones con mayores necesidades; la dimensión de comunicación ocupó el primer lugar $(\bar{X}=11,4 ; D E=0,15)$, seguida de la dimensión de atención personal $(\bar{X}=11,2 ; D E=0,12)$; con menor puntuación se encuentran las dimensiones de atención médica $(\bar{X}=10,3 ; D E=0,19)$ y posibles mejoras $(\bar{X}=7,6 ; D E=0,14)$ respectivamente. La puntuación global quedó representada por una media de datos $(\bar{X}=41,6 ; D E=1,8)$, como se muestra en la Tabla 3.

\section{DISCUSIÓN}

Las necesidades de los familiares con pacientes en condición crítica ha sido un fenómeno de interés para la comunidad científica en los últimos años, siendo diversos los estudios que han abordado esta problemática, así como la disponibilidad de instrumentos con adecuada calidad psicométrica ${ }^{(11,12)}$.

En este sentido, en Colombia, la literatura disponible da cuenta de abordajes interpretativos y otras veces orientado a la medición de la satisfacción general de la atención recibida (13,14); las investigaciones que utilizan escalas que permitan identificar la importancia dada por los familiares a necesidades asociadas a este proceso son escasa en el contexto Colombiano, y las que existen dan cuenta de un panorama de interés para enfermería, con susceptibilidad de continuidad investigativa ${ }^{(15)}$.

Referente a la caracterización sociodemográfica, lo descrito por Carreño y colegas; quienes encontraron que los cuidadores en las diferentes regiones de Colombia eran mujeres con edad promedio de 43 años, algún grado de escolaridad y en su gran mayoría el parentesco fue de hijo (a), situación que guarda similitud con los resultados obtenidos en esta investigación, manteniendo lo descrito en la literatura disponible, en relación a que la actividad de cuidado es realizada mayormente por mujeres (16-18). 
Por otra parte, el panorama de priorización de necesidades percibidas por los familiares estudiados da cuenta de que la información sincera relacionada con la condición de salud del paciente y los equipos que se están utilizando durante el proceso de atención revisten gran importancia para ellos, resultados que respaldan los hallazgos de diferentes autores, quienes han identificado la información como la necesidad más sentida por parte de los familiares de pacientes en $\mathrm{UCl}$, ya que estos esperan que se les informe sobre aspectos como los signos vitales, el cuidado recibido, el confort y el descanso del paciente, así como sobre el tratamiento y los aspectos concretos de las $\mathrm{UCl}$ como son el equipo tecnológico, dejando de lado otros elementos como la satisfacción de las necesidades propias e incluso aspectos del tratamiento médico $(19,20)$.

Siguiendo con este contexto, al evaluar estas necesidades por dominios, ocupa el primer lugar el dominio de comunicación, seguido del dominio de atención personal. Esto permite establecer la importancia que le otorgan los familiares a la relación con el personal de salud y que sean tenidos en cuenta en la toma de decisiones; situación que ha sido descrita como un elemento humanizador de la atención y adicional a ello, se ha demostrado su vínculo con la reducción en los días estancia (21-23).

Así mismo, se ha definido que el hecho de tener un integrante en una UCI supone para la familia una pérdida o modificación del rol que desempeñaba en el núcleo familiar y lo que se traduce en una crisis que el equipo de salud debe ayudar a solventar (24); en este sentido, el reconocimiento de la familia como parte importante de las acciones de cuidado permite minimizar los vacíos de información que genera esta experiencia.

\section{CONCLUSIONES}

En la presente investigación, la muestra estudiada se caracterizó por estar compuesta por mujeres, con un rango de en edad entre 50 a 69 años, con algún nivel de escolaridad; siendo la primaria completa el de mayor predominio, respecto al parentesco la mayoría refirió ser hijo.

Las necesidades en este grupo de familiares estuvieron enmarcadas en la comunicación con el equipo sanitario, así como el trato recibido por este; en este contexto, las necesidades asociadas a la atención médica y a mejoras en la infraestructura fueron infravaloradas.

El familiar de una persona ingresada en un servicio de cuidado intensivo debe ser tomado en cuenta en el proceso de atención, es claro que el eje de la atención deberá seguir siendo el paciente, sin embargo, es imperioso reconocer al familiar como un actor protagónico en los cuidados ofertados a la persona en condición crítica.

\section{REFERENCIAS}

1. Holanda Peña M.S. et al. Satisfacción en la Unidad de Cuidados Intensivos (UCI): la opinión del paciente como piedra angular. Med Intensiva [Internet]. 2017 [Citado 2020 abril 13]; 41(2):78---85. Disponible en: https://www.medintensiva.org/es- 
satisfaccion-unidad-cuidados-intensivos-uci--articulo-S0210569116301358

http://dx.doi.org/10.1016/j.medin.2016.06.007

2. Bautista Rodríguez Luz Marina, Arias Velandia María Fernanda, Carreño Leiva Zury Ornella. Percepción de los familiares de pacientes críticos hospitalizados respecto a la comunicación y apoyo emocional. Rev Cuid [Internet]. Diciembre 2016 [Citado: 2020 Abril 11]; $7 \quad$ (2):1297-1309. $\quad$ Disponible http://www.scielo.org.co/scielo.php?script=sci_arttext\&pid=S221609732016000200007 \&lng=enhttp://dx.doi.org/10.15649/cuidarte.v7i2.330

3. J.M. Velasco Bueno, A. Alonso-Ovies, G. Heras La Calle, C. Zaforteza Lallemand Main information requests of family members of patients in Intensive Care Units. Med Intensiva (English Edition). [Internet]. 2018 [Citado: 202010 abril]; 42 (6): 337 - 345.Disponible en: https://doi.org/10.1016/j.medin.2017.09.007

4. Padilla Fortunatti, Cristóbal \& Rojas, Noelia \& Amthauer-Rojas, M. \& Molina, Yerko. Necesidades de los familiares de pacientes críticos en un hospital académico de Chile. Enferm Intensiva [Internet], 2018 [Citado 10 abril 2020]; 29 (1): 32-40. Disponible enhttp://doi.org/10.1016/i.enfi.2017.09.001

5. A. Sánchez-Vallejo, D. Fernández, A. Pérez-Gutiérrez, M. FernándezFernández. Análisis de las necesidades de la familia del paciente crítico y la opinión de los profesionales de la unidad de cuidados intensivos. Med Intensiva, [Internet]. 2016 [Citado 10 abril 2020]; 40 (9): 527 - 540. Disponible en: https://doi.org/10.1016/j.medin.2016.03.005

6. M.I. Pardavila Belio, C.G. Vivar. Necesidades de la familia en las unidades de cuidados intensivos. Revisión de la literatura. Enferm Intensiva, [Internet]. 2012 [Citado 10 abril 2020]; 23(2):51-67.Disponible en: https://doi.org/10.1016/j.enfi.2011.06.001

7. Boada Quijano, Laura Carolina. Información: la principal necesidad de las familias en Unidad de Cuidados Intensivos (UCI). 2016 Tesis de Maestría Repositorio Universidad Nacional de Colombia, [Internet] 2016 [Citado 10 abril 2020]. Disponible en: http://bdigital.unal.edu.co/55973/7/LauraC.BoadaQuijano.2016.pdf

8. C. Padilla-Fortunatti, N. Rojas-Silva, M.C. Arechabala-Mantuliz. Análisis de la diferencia entre importancia y satisfacción de las necesidades de los familiares de pacientes críticos. Med Intensiva [Internet] 2019 [citado 9 abril 2020]; 43(4):217224.ISSN0210-5691. https://doi.org/10.1016/j.medin.2018.06.010

9. G. Martos-Casado, A. Aragón-López, N. Gutiérrez-Ramos. Satisfacción de los familiares de los pacientes ingresados en una unidad de cuidados intensivos: percepción de los familiares y los profesionales. Enferm Intensiva [Internet], 2014 [citado 9 abril 2020]; 25(4):164-72.Disponible http://doi.org/10.1016/j.enfi.2014.10.001

10. Gómez Martínez S., Ballester Arnal R., Gil Juliá B. El Cuestionario de Necesidades de los Familiares de Pacientes de Cuidados Intensivos (CCFNI) versión breve: adaptación y validación en población española. Anales Sis San Navarra [Internet]. 2011 Dic [citado 2020 Abr 05]; 34(3): 349-361. Disponible en: http://scielo.isciii.es/scielo.php?script=sci_arttext\&pid=S1137-

66272011000300002\&lng=eshttp://dx.doi.org/10.4321/S1137-66272011000300002.

11. Martínez Toquero J, Llauradó Serra M. Necesidades de los pacientes ingresados en la Unidad de Cuidados Intensivos. Metas Enferm dic [Internet] 2017 [citado 2020 Abr 05]; 19(10): 63-70. Disponible en: https://www.enfermeria21.com/revistas/metas/articulo/81010/

12. Olano M., Vivar C. G. Instrumentos para la valoración de las necesidades de los familiares de pacientes ingresados en cuidados intensivos: una revisión sistemática. Anales Sis San Navarra [Internet]. 2012 Abr [citado 2020 Abr 06]; 
http://scielo.isciii.es/scielo.php?script=sci_arttext\&pid=S1137-

66272012000100006\&lng=es. http://dx.doi.org/10.4321/S1137-66272012000100006.

13. Márquez Herrera, Marisela, Carrillo González, Gloria Mabel, La experiencia del familiar de la unidad de cuidados intensivos en Bucaramanga (Colombia): un estudio fenomenológico. Archivos de Medicina (Col) [Internet]. 2015 [citado 2020 Abr 06]; 15(1):95-106. Disponible en: https://www.redalyc.org/articulo.oa?id=273840435010

14. Bautista Rodríguez Luz Marina, Arias Velandia María Fernanda, Carreño Leiva Zury Ornella. PERCEPCIÓN DE LOS FAMILIARES DE PACIENTES CRÍTICOS HOSPITALIZADOS RESPECTO A LA COMUNICACIÓN Y APOYO EMOCIONAL. Rev Cuid [Internet]. 2016 [Citado: 2020 Abril 05]; 7(2): 1297-1309. Disponible en: http://www.scielo.org.co/scielo.php?script=sci_arttext\&pid=S2216-

$09732016000200007 \&$ Ing=en. http://dx.doi.org/10.15649/cuidarte.v7i2.330.

15. Galvis López CR, Salamanca Ramos E. Percepción de necesidades en cuidadores familiares de adultos internados en una unidad de cuidados intensivos de una institución prestadora de salud (IPS) privada en Villavicencio, Colombia. Investig Enferm. Imagen Desarr [Internet]. 2014 [Citado: 2020 Abril 05]; 16(2):81-94. Disponible en: http://dx.doi:10.11144/Javeriana.IE16-2.pncf

16. Carreño Moreno S, Chaparro Díaz O. Agrupaciones de cuidadores familiares en Colombia: perfil, habilidad de cuidado y sobrecarga. Pensam. psicol. [Internet]. 17ene.2017 [citado 8 abr.2020]; 15(1). Disponible en: https://revistas.javerianacali.edu.co/index.php/pensamientopsicologico/article/view/139 $\underline{3}$

17. Jiménez Ruiz Ismael, Moya Nicolás María. La cuidadora familiar: sentimiento de obligación naturalizado de la mujer a la hora de cuidar. Enferm. glob. [Internet]. 2018 [citado 2020 Abr 08]; 17(49): 420-447. Disponible en: http://scielo.isciii.es/scielo.php?script=sci_arttext\&pid=\$1695-

61412018000100420\&lng=es. Epub 01-Ene-2018. http://dx.doi.org/10.6018/eglobal.17.1.292331.

18. Bello Carrasco, Lourdes Maribel, León Zambrano, Génesis Andreina, \& Covena Bravo, María Isabel. (2019). Factores que predominan sobrecarga en el cuidador formal e informal geriátrico con déficit de autocuidado. Revista Universidad y Sociedad [Internet] 2019 [citado 2020 Abr 08]; 11(5), 385-395. Disponible en: http://scielo.sld.cu/scielo. php?script=sci arttext\&pid=S2218-

36202019000500385\&lng=es\&tlng=es.

19. Pardavila Belio M, Vivar C.G. Necesidades de la familia en las unidades de cuidados intensivos. Revisión de la literatura. Enferm Intensiva. [Internet]. 2012 [citado $2020 \mathrm{Abr}$ 08]; 23(2):51-67. Disponible en: https://www.elsevier.es/es-revistaenfermeria-intensiva-142-articulo-necesidades-familia-unidades-cuidados-intensivos-S1130239911000460

20. Boada Quijano L, Guáqueta Parada S. Elementos para abordar la necesidad de información de las familias en una unidad de cuidados intensivos. IEID [Internet]. 4dic.2019 [citado 7abr.2020]; 21(2). Disponible en: https://revistas.javeriana.edu.co/index.php/imagenydesarrollo/article/view/21474

21. Bautista Rodríguez Luz Marina, Arias Velandia María Fernanda, Carreño Leiva Zury Ornella. PERCEPCIÓN DE LOS FAMILIARES DE PACIENTES CRÍTICOS HOSPITALIZADOS RESPECTO A LA COMUNICACIÓN Y APOYO EMOCIONAL. Rev Cuid [Internet]. 2016 [citado abril 07 2020]; 7(2): 1297-1309. Disponible en: http://www.scielo.org.co/scielo.php?script=sci arttext\&pid=S2216-

09732016000200007\&lng=enhttp://dx.doi.org/10.15649/cuidarte.v7i2.330. 
22. Lee, H.W., Park, Y., Jang, E.J. et al. Intensive care unit length of stay is reduced by protocolized family support intervention: a systematic review and metaanalysis. Intensive Care Med [Internet]. 20192016 [citado abril 07 2020]; 45 (8): 1072-1081

Disponible en:

https://www.ncbi.nlm.nih.gov/pubmed/31270579https://doi.org/10.1007/s00134-01905802-y

23. De La Cueva Ariza et al. Implementation of the evidence for the improvement of nursing care to the critical patient's family: a Participatory Action Research. BMC Health Serv Res. [Internet] 2018 [citado abril 07 2020]; 18 (1): 357. Disponible en: https://www.ncbi.nlm.nih.gov/pmc/articles/PMC5946428/https://doi.org/10.1186/s1291 3-018-3177-8

24. Aliberch Raurell A.M, Miquel Aymar I.M. Necesidad de rol en los familiares del paciente en la unidad de cuidados intensivos. Enferm Intensiva [Internet]. 2015[citado abril 07 2020]; 26(3):101-111. Disponible en: https://www.elsevier.es/es-revistaenfermeria-intensiva-142-articulo-necesidad-rol-familiares-del-pacienteS1130239915000310http://dx.doi.org/10.1016/j.enfi.2015.02.001 